Pacific Journal of Mathematic 


\title{
TOPOLOGICAL LATTICE ORDERED GROUPS
}

\author{
RICHARD N. BALL
}

\begin{abstract}
Several types of hulls and completions of lattice ordered groups have been obtained by algebraic methods. In this paper is laid some groundwork for the application of topological and uniform-space concepts to the same end by setting forth those links-topological, algebraic and semanticbetween a topological lattice ordered group $H$ and a topologically dense $\ell$-subgroup $G$.
\end{abstract}

In section one a convex $\ell$-subgroug of a representable $\ell$-group $G$ is proved to be order closed if and only if it is closed with respect to every Hausdorff $\ell$-topology. In section three the disjunctive formulas which hold in a topological $\ell$-group are proved to be the same as those which hold in a topologically dense $\ell$-subgroup. The last section contains the continuous versions of the classical $\ell$-group representation theorems.

The list of contributors to the theory of topological lattice ordered groups is long; Redfield gives a historical sketch and a good bibliography in [18]. This investigation makes particularly heavy use of the work of R. H. Madell, that of Redfield, and the ongoing work of B. Smarda. But for G. Otis Kenny, whose work introduced the author to these ideas and who unselfishly participated in many stimulating discussions, and for Stephen H. McCleary, whose penetrating comments improved an earlier version, the author reserves his deepest gratitude.

1. Order aud topological closure. A topology $\mathscr{T}$ on a lattice ordered group $G$ which makes group and lattice operations continuous will be termed an $\ell$-topology. $(G, \mathscr{T})$ is a topological lattice ordered group or t $\ell$-group. Smarda [20] first characterized an $\ell$ topology in terms of the neighborhood filter of the identity.

THEOREM 1.1. If $\mathscr{B}$ is the neighborhood filter of 1 of the ttgroup $(G, \mathscr{T})$, then $\mathscr{B}$ satisfies the following conditions.

(a) $\mathscr{B}$ is a normal filter of subsets of $G$ each containing 1.

(b) If $B \in \mathscr{B}$ then $B^{-1} \in \mathscr{B}$.

(c) If $B \in \mathscr{B}$ then there is some $A \in \mathscr{B}$ such that $A \cdot A \subseteq B$.

(d) If $B \in \mathscr{B}$ and if $a$ and $b$ are disjoint members of $G$ then there is some $A \in \mathscr{B}$ with $A a \wedge A b \subseteq B$.

The neighborhood filter of any $g \in G$ is $\mathscr{B} g=g \mathscr{B}$. Conversely, if $\mathscr{B}$ is any filter of subsets of $G$ satisfying (a) (d) then by defining 
the neighborhood filter of each $g \in G$ to be $\mathscr{B} g$ one obtains the unique $\ell$-topology on $G$ having $\mathscr{B}$ as its neighborhood filter of 1 . $\mathscr{T}$ is Hausdorff if and only if $\cap \mathscr{B}=1$.

On account of the preceding theorem we shall often blur the distinction between $\mathscr{T}$ and $\mathscr{B}$, saying, for example, "let $\mathscr{B}$ be an $\ell$-topology," or "the $t \ell$-group $(G, \mathscr{B}) . "$

A subset $X$ of an $\ell$-group $G$ is convex if $x_{1} \leqq g \leqq x_{2}$ and $x_{1}, x_{2} \in$ $X$ imply $g \in X$. $\quad X^{\sim}$ designates $\left\{g \in G \mid x_{1} \leqq g \leqq x_{2}, x_{1}, x_{2} \in X\right\}$, the convexification of $X$ in $G$. A subset $X$ of an $\ell$-group $G$ is order closed if $g \in X$ whenever $S \subseteq X$ and $g=\bigvee S$ or $g=\bigvee S$. The smallest order closed subset of $G$ containing an arbitrary subset $X$ is designated $\operatorname{ocl}_{G}(X)$ or simply ocl $(X)$. One may describe ocl $(X)$ inductively as follows. Let $X_{0}$ be $X$, let $X_{r+1}$ be $\{g \in G \mid g=\Lambda S, S \subseteq$ $\left.X_{\gamma}\right\}$ for $\gamma$ even, let $X_{\gamma+1}$ be $\left\{g \in G \mid g=\Lambda S, S \subseteq X_{r}\right\}$ for $\gamma$ odd, and let $X_{r}=U\left\{X_{\delta} \mid \delta<\gamma\right\}$ for limit ordinals $\gamma$. Then ocl $(X)=X_{\eta}$, where $\eta$ is some ordinal such that $X_{\eta}=X_{\eta+1}=X_{\eta_{+2}}$. The reader should be aware that the order closure operator is never topological in the sense of Kuratowski since ocl $\{a, b\}=\{a, b, a \vee b, a \wedge b\} \neq\{a, b\}=$ ocl $\{a\} \cup$ ocl $\{b\}$. However, the order closure operator may be topological on sublattices or on convex sublattices, as we shall see. In any case, a better understanding of the interplay between order and topological closure must be a central issue in the theory of $t \ell$-groups.

The next lemma has a straightforward inductive proof.

Lemma 1.2. If $A$ and $B$ are subsets and $g$ an element of an l-group, then

(a) ocl $(g A)=g \cdot o c l(A)$,

(b) $\operatorname{ocl}\left(A^{-1}\right)=\operatorname{ocl}(A)^{-1}$,

(c) $\operatorname{ocl}(A) \cdot \operatorname{ocl}(B) \subseteq \operatorname{ocl}(A B)$,

(d) ocl $(A) \vee \operatorname{ocl}(B) \subseteq \operatorname{ocl}(A \vee B)$ and dually.

CoRollary 1.3. If $A$ is a subgroup, a normal subgroup, or a sublattice of some $\ell-g r o u p$, then so is ocl $(A)$.

The order closure of a convex set need not be convex. However, the order closure of a convex sublattice is a convex sublattice.

Proposition 1.4. Suppose $G$ is an l-subgroup of the $\ell$-group $H$ and that $X$ is a convex sublattice of $G$. Then $\operatorname{ocl}_{H}(X)$ is a convex sublattice of $\operatorname{ocl}_{H}(G)$.

Proof. It is clear that each $G_{\gamma}$ and each $X_{\gamma}$ is a sublattice of 
$H$. It is necessary only to show that if $X_{\gamma}$ is convex in $G_{\gamma}$ then $X_{\gamma+1}$ is convex in $G_{\gamma+1}$. If $\mathrm{V} x_{\alpha} \leqq \mathrm{V} g_{\beta} \leqq \mathrm{V} x_{\dot{\delta}}$ for $x_{\alpha}, x_{i} \in X_{\gamma}$ and $g_{\beta} \in G_{r}$, it follows that for particular $\alpha, \beta$ and $\delta, x_{\alpha} \leqq x_{\alpha} \vee\left(g_{\beta} \wedge x_{\delta}\right) \leqq$ $x_{\alpha} \vee x_{i}$. Since all of these objects must be in $X_{r}$, we have $\mathrm{V} g_{\beta}=\mathrm{V}$ $\left(x_{\alpha} \vee\left(g_{\beta} \wedge x_{i}\right)\right) \in X_{\gamma+1}$.

The topological closure operator, written $\operatorname{cl}(X)$ for $X$ a subset of a $t /$-group, satisfies the analogues of the previous two propositions. But a lemma is necessary first.

Lemma 1.5. Suppose $X$ is a sublattice of the Hausdorff t/-group $H$. For $h_{1}, h_{2} \in \operatorname{cl}(X)$ the following are equivalent:

(a) $h_{1} \geqq h_{2}$.

(b) For any neighborhoods $N_{1}$ and $N_{2}$ of $h_{1}$ and $h_{2}$ respectively there is a neighborhood $V$ of $h_{1}$ with $V \cong N_{1}$ such that for any $x_{1} \in V \cap X$ there is some $x_{2} \in N_{2} \cap X$ with $x_{1} \geqq x_{2}$.

(c) For any neighborhoods $N_{1}$ and $N_{2}$ of $h_{1}$ and $h_{2}$ respectively there is some neighborhood $V$ of $h_{2}$ with $V \cong N_{2}$ such that for any $x_{2} \in V \cap X$ there is some $x_{1} \in N_{1} \cap X$ with $x_{1} \geqq x_{2}$.

(d) For any neighborhoods $N_{1}$ and $N_{2}$ of $h_{1}$ and $h_{2}$ respectively there are elements $x_{1} \in N_{1} \cap X$ and $x_{2} \in N_{2} \cap X$ such that $x_{1} \geqq x_{2}$.

Proof. (a) implies (b). Suppose $N_{1}$ and $N_{2}$ are neighborhoods of $h_{1}$ and $h_{2}$ respectively, and that $h_{1} \geqq h_{2}$. By the continuity of $\wedge$ there are neighborhoods $V$ and $U$ of $h_{1}$ and $h_{2}$ such that $V \wedge U \subseteq N_{2}$ and $V \cong N_{1}, U \subseteq N_{2}$. Fix $x \in U \cap X$. Then for any $x_{1} \in V \cap X$ let $x_{2}=x_{1} \wedge x \in N_{2} \cap X$. (a) implies (c) is similar, while (b) implies (d) and (c) implies (d) are clear. Finally, to show that (d) implies (a), consider neighborhoods $A$ and $B$ of $h_{1} \wedge h_{2}$ and $h_{2}$ respectively. Let $N_{1}$ and $N_{2}$ be neighborhoods of $h_{1}$ and $h_{2}$ such that $N_{1} \wedge N_{2} \subseteq A$ and $N_{2} \subseteq B$. If $x_{1} \in N_{1} \cap X$ and $x_{2} \in N_{2} \cap X$ satisfy $x_{1} \geqq x_{2}$, then $x_{2}=x_{1} \wedge x_{2} \in A \cap B \neq \phi$. Since $H$ is Hausdorff, $h_{1}=h_{1} \wedge h_{2}$.

An easy refinement of the preceding argument establishes this corollary.

CoRollary 1.6. Suppose $X$ is a sublattice of the Hausdorff t८-group $H$ and that $h_{1}, h_{2}, h_{3} \in \operatorname{cl}(X)$. Then $h_{1} \leqq h_{2} \leqq h_{3}$ if and only if for all neighborhoods $N_{1}, N_{2}$ and $N_{3}$ of $h_{1}, h_{2}$ and $h_{3}$ respectively, there are elements $x_{i} \in X \cap N_{i}$ with $x_{1} \leqq x_{2} \leqq x_{3}$. Furthermore, if one or more of the $h_{i}$ 's happen to be in $X$, the $x_{i}$ corresponding to that $h_{i}$ may be chosen to be $h_{i}$.

The positive cone of an $/$-group $G$, written $G^{+}$, is $\{g \in G \mid g \geqq 1\}$. 
COROLLARY 1.7. For an $\ell$-subgroup $G$ of the Hausdorff $t \ell-g r o u p$ $H, \operatorname{cl}(G)^{+}=\operatorname{cl}\left(G^{+}\right)$.

The first assertion of the next proposition is due to Smarda ([20], Theorem 3.5), while the second assertion slightly generalizes a result of Madell ([16], Proposition 4). A prime subgroup is a convex $\ell$-subgroup which contains at least one element of each disjoint pair of elements.

Proposition 1.8. Suppose $(H, \mathscr{B})$ is a t/-group. If $X$ is a subgroup, a normal subgroup, or a sublattice then so is $\operatorname{cl}(X)$. If $G$ is a sublattice of $H$ and $X$ is a convex sublattice of $G$ then $\mathrm{cl}(X)$ $i s$ a convex sublattice of $\mathrm{cl}(G)$. If $X$ is a prime subgroup of $G$ then $\mathrm{cl}(X)$ is a prime subgroup of $\mathrm{cl}(G)$.

Proof. The first statement is a direct result of the continuity of the operations. Suppose that $X$ is a convex sublattice of the sublattice $G$ of $H$ and that $y_{1} \leqq h \leqq y_{2}$ for $y_{1}, y_{2} \in \operatorname{cl}(X)$ and $h \in \operatorname{cl}(G)$. For $U$ an arbitrary neighborhood of $h$ find neighborhoods $Y$ and $V$ of $y_{2}$ and $h$ such that $Y \wedge V \cong U$. By Lemma 1.5 there is a neighborhood $W$ of $y_{1}$ such that for any $g \in W \cap G$ there is some $g^{\prime} \in V \cap G$ with $g \leqq g^{\prime}$. Finally, choose neighborhoods $N_{1}$ and $N_{2}$ of $y_{1}$ and $y_{2}$ respectively such that $N_{1} \wedge N_{2} \subseteq W$. Letting $x_{1} \in N_{1} \cap X$ and $x_{2} \in N_{2} \cap Y \cap X$ we get $x_{1} \wedge x_{2} \in W \cap X$. Hence there exists $g_{0} \in V \cap G$ with $x_{1} \wedge x_{2} \leqq g_{0}$. Then $x_{1} \wedge x_{2} \leqq g_{0} \wedge x_{2} \leqq x_{2}$, which implies $g_{0} \wedge x_{2} \in U \cap X$. That is, $h \in \operatorname{cl}(X)$. (The part of Lemma 1.5 used in this argument does not require $\mathscr{B}$ to be Hausdorff.) Finally, suppose $X$ is a prime subgroup of $G$ and that $h_{1}$ and $h_{2}$ are disjoint members of $\mathrm{cl}(G)$. If $h_{1} \notin \mathrm{cl}(X)$ there must be a neighborhood $N_{1}$ of $h_{1}$ disjoint from $X$. If $N_{2}$ is any neighborhood of $h_{2}$ then, since $\left(N_{1} \cap G\right) \wedge\left(N_{2} \cap G\right)$ is a neighborhood of 1 in $G$, there are elements $g_{1} \in N_{1} \cap G$ and $g_{2} \in N_{2} \cap G$ with $g_{1} \wedge g_{2}=1$. This forces $g_{2} \in X$ and $h_{2} \in \operatorname{cl}(X)$.

Two types of $\ell$-topologies assume particular importance in what follows. As $\ell$-topology $\mathscr{B}$ is said to be convex if $\mathscr{B}$ has a base of convex sets and order closed if $\mathscr{B}$ has a base of order closed sets. The next proposition can be easily established using Lemma 1.2 .

Proposition 1.9. Given an $<$-topology $\mathscr{B}$ let $\mathscr{B} \sim$ be the filter generated by sets $B^{\sim}$ for $B \in \mathscr{B}$ and let ocl $(\mathscr{B})$ be the filter generated by sets ocl $(B)$ for $B \in \mathscr{B}$. Then $\mathscr{B}^{\sim}$ is the finest convex $\iota$-topology coarser than $\mathscr{B}$ and ocl $(\mathscr{B})$ is the finest order closed 
¿-topology coarser than $\mathscr{B}$.

Redfield [18] first noticed that if $\mathscr{B}$ is a filter having a base of convex sets then requirement (d) of Theorem 1.1 could be replaced by the following weaker condition.

(d') If $B \in \mathscr{B}$ then there is some $A \in \mathscr{B}$ with $A \wedge A \subseteq B$.

When verifying that a filter of subsets is an $\ell$-topology we shall use $\left(d^{\prime}\right)$ whenever it is clear that the filter has a base of convex sets.

Not every $\ell$-topology is convex, of course, but a rather intimate relationship between $\mathscr{B}$ and $\mathscr{B}^{\sim}$ exists. For example, $\mathscr{B}$ is Hausdorff if and only if $\mathscr{B}^{\sim}$ is Hausdorff. These results are due to Smarda [19].

Proposition 1.10. For any $\ell$-topology $\mathscr{B}$ on an $\ell$-group $G$, $\cap \mathscr{B}=\cap \mathscr{B}^{\sim}$.

Proof. Consider $1<x \in \cap \mathscr{B}^{\sim}$ and an arbitrary $B \in \mathscr{B}$. Since $x \wedge 1=1$ there must be some $A \in \mathscr{B}$ with $A x \wedge A \subseteq B$. But $x \in A^{\sim}$ implies $x \leqq a$ for some $a \in A$. Therefore $x=x \wedge a \in A x \wedge A \leqq B$. That is, $x \in \cap \mathscr{B}$.

An $\ell$-ideal is a normal convex $\ell$-subgroup of an $\ell$-group. $\ell$ ideals are the kernels of $\ell$-homomorphisms.

CoROLlaRy 1.11. If $\mathscr{B}$ is any l-topology on the l-group $G$ then $\cap \mathscr{B}$ is an $\ell$-ideal of $G$.

The next proposition provides a link between topological and order closures of an $\ell$-subgroup. It implies that for $G$ an $\ell$-subgroup of the Hausdorff $t \ell$-group $H, \mathrm{cl}(G)$ is an $\ell$-subgroup of ocl $\left(G^{\sim}\right)$. This rules out, for example, the possibility that $\mathrm{cl}(G)$ ever be a lex extension of $G$.

Proposition 1.12. Suppose $G$ is an 6 -subgroup of the Hausdorff t $\ell$-group $(H, \mathscr{B})$. Then for any $h \in \mathrm{cl}(G)$ and any neighborhood $N$ of $h, h=\bigvee\{h \wedge g \mid g \in N \cap G\}=\Lambda\{h \vee g \mid g \in N \cap G\}$ in $\operatorname{cl}(G)$.

Proof. Suppose that for some $k \in \mathrm{cl}(G), k \geqq h \wedge g$ for all $g \in N \cap$ $G$. Given neighborhoods $A$ and $B$ of $h$ and $k$ respectively let $C$ be a neighborhood of $h$ satisfying $C \subseteq A$ and $C \wedge C \subseteq A$. Choosing $k \in B$ and $g \in C \cap N \cap G$ gives $k \geqq g \wedge h \in A$. If the $X$ of Lemma $1.5(\mathrm{~d})$ is taken to be $H$ we get $k \geqq h$. 
The next proposition implies, among other things, that $\mathrm{cl}(G)$ is never a lex extension of $G$.

COROLLARY 1.13. If $G$ is a subgroup of the Hausdorff t/-group $H$ then $\operatorname{cl}(G)$ is an $\ell$-subgroup of ocl $\left(G^{\sim}\right)$.

One of the reasons for studying order closed $\ell$-topologies on an $\ell$-subgroup $G$ of a $t \ell$-group $H$ is the particularly close connection between $G$ and $\mathrm{cl}(G)$ which results in this case. To say that $G$ is order dense in $H$ is to say that for every $1<h \in H$ there is some $g \in G$ such that $1<g \leqq h$. It is well known that this condition implies that suprema and infima in $G$ and $H$ agree.

Proposition 1.14. Suppose $G$ is an $/$-subgroup of the Hausdorff t८-group $(H, \mathscr{B})$. If $\mathscr{B}$ is an order closed $\ell$-topology on $G$, then $G$ is order dense in $\mathrm{cl}(G)$.

Proof. Consider $1<h \in \operatorname{cl}(G)$. Let $J$ be some neighborhood of $h$ such that $1 \notin J$ and $J \cap G$ is order closed in $G$. Let $S$ be $J \cap G^{+}$, a nonempty set by Corollary 1.7. There must be some $g \in G$ with $1<g \leqq S$, for otherwise $\Lambda S=1$, contrary to assumption. By Corollary $1.6, g \leqq h$.

The point of the following example is that, in the notation of Proposition 1.14, $\mathscr{B}$ may be order closed on $\mathrm{cl}(G)$ without being order closed on $G$. Let $H$ be $\boldsymbol{R}^{R}$, the $\ell$-group of all real-valued functions on the real numbers $\boldsymbol{R}$ with componentwise group and lattice operations (cardinal order). Let $G$ be $C(R)=\{h \in H \mid h$ continuous $\}$. Let $\mathscr{B}$ be the Hausdorff $\ell$-topology on $H$ generated by the prime subgroups $H_{r}=\{h \in H \mid(r) h=0\}$ for $r \in \boldsymbol{R}$. The reader may verify that $\mathscr{B}$ is not order closed on $G$ but is order closed on $H$, which is the closure of $G$ with respect to $\mathscr{B}$. An interesting open question is whether $\mathscr{B}$ must be order closed on $\mathrm{cl}(G)$ whenever $\mathscr{B}$ is order closed on $G$.

The following proposition is a slight generalization of a result of Madell ([16], Theorem 9).

THEOREM 1.15. An order closed convex sublattice $X$ of an $\ell-$ group $G$ is closed with respect to any Hausdorff l-topology on $G$.

Proof. Suppose $G$ is endowed with some Hausdorff $\ell$-topology and $y \in \operatorname{cl}(X)$.

Case 1. $y \geqq x_{0}$ for some $x_{0} \in X$. Then $y_{0} \in\left(\operatorname{cl}(X) \vee x_{0}\right) \wedge y \subseteq$ 
$\operatorname{cl}\left(\left(X \vee x_{0}\right) \wedge y\right)=\operatorname{cl}(K)$, where $K=\left\{x \in X \mid x_{0} \leqq x \leqq y\right\}$. Now $y=\bigvee K$, for if $K \leqq z<y$, then $\{g \in G \mid g \not z\}$ is an open subset of $G$ containing $y$ disjoint from $K$. Here we make use of the fact that $\{g \in$ $G \mid g \leqq z\}$ is closed, being the inverse image of the closed set. $\{z\}$ under the continuous map $g \rightarrow g \vee z$. Therefore $y \in X$.

Case 2. $y \leqq x_{0}$ for some $x_{0} \in X$. Similar to Case 1 .

Case 3. $y$ is unrelated to any member of $X$. Fix $x_{0} \in X$. Since $\mathrm{cl}(X)$ is a sublattice, $y \vee x_{0}$ and $y \wedge x_{0}$ are in $\mathrm{cl}(X)$. By Cases 1 and $2, y \vee x_{0}$ and $y \wedge x_{0}$ are in $X$. By the convexity of $X, y \in X$.

Is any order closed $\ell$-subgroup $X$ of the $\ell$-group $G$ closed with respect to every Hausdorff $\ell$-topology on $G$ ? An affirmative answer to this question would have interesting repercussions and makes the relaxation of the convexity hypotheses of Theorem 1.15 an important open question.

The remainder of this section is devoted to proving several near converses to Theorem 1.15 and to exploring the consequences. The utility of these partial converses lends importance to the search for a full converse. In order to prove any converse one must construct, for a given sublattice $X$ and given $y=\mathrm{V} S$ for $S \subseteq X$, a Hausdorff $\ell$-topology $\mathscr{B}$ with respect to which $y \in \operatorname{cl}(X)$. The next lemma provides a hint as to how this can be done.

A subset $T \leqq G^{+}$is a filter if $t_{1}, t_{2} \in T$ implies $t_{1} \wedge t_{2} \in T$ and if $g \geqq t \in T$ implies $g \in T$. If $T \subseteq G^{+}$has only the first property then we often blur the distinction between $T$ and the filter it generates, $\{g \in G \mid g \geqq t$ for some $t \in T\}$. For example, by $T^{n}$ is meant the filter $\left\{g \in G \mid g \geqq t^{n}\right.$ some $\left.t \in T\right\}, n$ a positive integer. If $T$ is a filter on $G^{+}$let us agree to term an $\ell$-topology $\mathscr{B}$ T-coarse if every $B \in \mathscr{B}$ intersects $T$; that is, if $1 \in \mathrm{cl}(T)$. Several points deserve mention. First, if $\mathscr{B}$ is $T$-coarse then $\mathscr{B}$ is also $g^{-1} T^{n} g$-coarse for each $g \in G$ and each positive integer $n$. Secondly, if $\mathscr{B}$ is Hausdorff and $T$-coarse then $\Lambda T=1$. Thirdly, if $\mathscr{B}$ is $T$-coarse and $\mathscr{C}$ is a coarser $\ell$-topology than $\mathscr{B}$ then $\mathscr{C}$ is also $T$-coarse.

Proposition 1.16. Suppose $X$ is a sublattice of the t/-group $(H, \mathscr{B})$ and that $S=\{x \in X \mid x \leqq y\} \neq \phi$. Then $y \in \operatorname{cl}(S)$ if and only if $\mathscr{B}$ is $y S^{-1}$-coarse.

Proof. $\mathscr{B}$ is $y S^{-1}$-coarse if and only if $1 \in \mathrm{cl}\left(y S^{-1}\right)$ if and only if $y \in \operatorname{cl}(S)$.

Given a sublattice $X$ and $y=\bigvee S$ for some $S \subseteq X$, let $T=y S^{-1}$. To form a Hausdorff convex $\ell$-topology $\mathscr{B}$ which is $T$-coarse we 
need only decide, for each $n$ and $g$, which member of $g^{-1} T^{n} g$ to include in each $B \in \mathscr{B}$. But first an observation which plays a technical role in the proof. A subset $F \cong G$ is upper directed if for any $f_{1}, f_{2} \in F$ there is some $f_{3} \in F$ with $f_{3} \geqq f_{1}, f_{2}$.

Lemma 1.17. Suppose $g=\bigvee F$ for some upper directed subset $F$ of the $\ell$-group $G$. Then $g^{n}=\bigvee\left\{f^{n} \mid f \in F\right\}$.

$$
\text { Proof. } \begin{aligned}
g^{2} & =\mathrm{V}\left\{f_{1} \mid f_{1} \in F\right\} \cdot \bigvee\left\{f_{2} \mid f_{2} \in F\right\}=\mathrm{V}\left\{f_{1} f_{2} \mid f_{i} \in F\right\} \\
& =\mathrm{V}\left\{\left(f_{3}\right)^{2} \mid f_{3} \geqq f_{1}, f_{2} ; f_{3} \in F\right\} .
\end{aligned}
$$

Conrad attributes the archimedean case of the next corollary to Bernau [5]. An /-group is representable if its minimal prime subgroups are normal.

COROLLARY 1.18. An l-group $G$ is representable if and only if $F \subseteq G$ and $g=\bigvee F$ imply $g^{n}=\bigvee\left\{f^{n} \mid f \in F\right\}$.

Proof. Suppose $G$ is representable, $F \subseteq G$, and $g=\bigvee F$. Since $F^{\prime}=\left\{f_{1} \vee f_{2} \vee \cdots \vee f_{k} \mid f_{i} \in F\right\}$ is upper directed, $g^{n}=\bigvee\left\{f^{n} \mid f \in F^{\prime}\right\}$. But if $G$ is representable then $\left(f_{1} \vee f_{2} \vee \cdots \vee f_{k}\right)^{n}=\left(f_{1}\right)^{n} \vee\left(f_{2}\right)^{n} \vee \cdots$ $\vee\left(f_{k}\right)^{n}$, so $g^{n}=\bigvee\left\{f^{n} \mid f \in F\right\}$.

THEOREM 1.19. If $T \cong G^{+}$is a normal filter on the representable $\ell$-group $G$ and $\Lambda T=1$, then there is a convex Hausdorff ८-topololy $\mathscr{B}$ which is T-coarse.

Proof. The reader should note that each $T^{n}$ is a normal filter, that $\Lambda T^{n}=1$, and that $T \supseteqq T^{2} \supseteqq T^{3} \supseteqq \cdots$. For each map $f: N \rightarrow G^{+}$ such that $(n) f \in T^{n}$ define $B_{f}=\{x \in G|| x \mid \leqq(1) f \vee(2) f \vee \cdots \vee(n) f$ some $n \in N\}$. Let $\mathscr{B}$ be the filter generated by all such $B_{f}$ 's. We claim that $\mathscr{B}$ is a Hausdorff $\ell$-topology. Note that $\mathscr{B}$ is convex and $T$-coarse by definition.

(a) $\mathscr{B}$ is a filter since, given $f: N \rightarrow G^{+}$and $k: N \rightarrow G^{+}$with $(n) f \in T^{n}$ and $(n) k \in T^{n}$ we have $B_{f} \cap B_{k} \supseteqq B_{h}$ where $(n) h=(n) f$ $\wedge(n) k$.

(b) Clearly $B_{f}^{-1}=B_{f}$ and $B_{f} \vee B_{f}=B_{f} \wedge B_{f}=B_{f}$.

(c) Given $B_{f} \in \mathscr{B}$ define $k: N \rightarrow G^{+}$so that $(n) k \in T^{n}$ satisfies $((n) k)^{2} \leqq(2 n) f \in T^{2 n}$. Then $[(1) k \vee(2) k \vee \cdots \vee(n) k]^{2}=((1) k)^{2} \vee((2) k)^{2} \vee \cdots$ $\vee((n) k)^{2} \leqq(2) f \vee(4) f \vee \cdots \vee(2 n) f \leqq(1) f \vee(2) f \vee \cdots \vee(2 n) f$. Therefore $B_{k} \cdot B_{k} \subseteq B_{f}$.

(d) To show $\mathscr{B}$ Hausdorff consider $1<x \in G$. Define (1) $f \in T$ 
so that (1) $f \geqq x$, possible since $\bigwedge T=1$. Define $(2) f \in T^{2}$ so that (1) $f \vee(2) f \geqq x$, possible since (1) $f=\Lambda\left\{t \vee(1) f \mid t \in T^{2}\right\}$. The function $f$ defined by proceeding in this fashion gives $B_{f} \in \mathscr{B}$ with $x \notin B_{f}$.

Corollary 1.20. For a convex sublattice $X$ of an abelian $\ell-$ group $G$ the following are equivalent.

(a) $X$ is order closed.

(b) $X$ is closed with respect to every Hausdorff $\ell$-topology.

(c) $X$ is closed with respect to every convex Hausdorff $\ell$-topology.

If the filter $T$ of Theorem 1.19 has the property that $T^{2}=T$ then it is the cone of a compatible tight Riesz order (CTRO), and $\mathscr{B}$ is generated by sets of the form $\left[t^{-1}, t\right]=\left\{g \in G \mid t^{-1} \leqq g \leqq t\right\}$. One may view such topologies as the most direct nontrivial generalization to $\ell$-groups of the interval topology on totally ordered groups. In these terms the topology constructed in Theorem 1.19 may be viewed as the next most direct such generalization. Furthermore, the proof of Corollary 1.20 asserts that if a convex sublattice of an abelian $\ell$-group fails to be closed with respect to any Hausdorff $\ell$-topology then it fails to be closed with respect to a topology of the sort used in Theorem 1.19. This fact argues in favor of the existence of many such topologies.

The preceding theorem can also be gotten for certain important types of nonnormal filters $T$. Let us agree to say that a filter $T$ on the representable $\iota$-group $G$ converges strongly to 1 if for every $g>1$ there is a normal prime subgroup $P$ omitting $g$ such that $\Lambda\{P t \mid t \in T\}=P$.

THeOREM 1.21. If $G$ is representable and $T$ is a filter on $G$ which converges strongly to 1 then there is a convex Hausdorff $\ell-$ topology $\mathscr{B}$ which is T-coarse.

Proof. For each $f: N \times G \rightarrow G^{+}$such that $(n, g) f \in g^{-1} T^{n} g$ define $B_{f}=\{x|| x \mid \leqq \bigvee(F) f$ for $F$ a finite subset of $N \times G\}$ and define $\mathscr{B}$ to be the filter generated by all such $B_{f}$ 's. As before, $\mathscr{B}$ is a $T$-coarse convex $\ell$-topology. To show $\mathscr{B}$ Hausdorff consider $1<x \in$ $G$ and let $P$ be the normal prime subgroup omitting $x$ such that $P=\Lambda\{P t \mid t \in T\}$. It follows that $P=\Lambda\left\{P g^{-1} t^{n} g \mid t \in T\right\}$ for every $n \in N$ and $g \in G$. Define $(n, g) f$ to be any member of $g^{-1} T^{n} g$ such that $P(n, g) f<P x$. Then for any finite subset $F \cong N \times G, P(\mathrm{~V}$ $(F) f)<P x$. Hence $\bigvee(F) f \geqq x$. Therefore $x \notin B_{f}$ and $\mathscr{B}$ is Hausdorff. 
LEMMA 1.22. If $C$ is any convex $<$-subgroup of the representable $\ell$-group $G$ and if $1<x \in \operatorname{ocl}(C)$, then for every $y>1$ there is a normal prime $P$ omitting $y$ such that $P c=P x$ for some $c \in C$, $c \leqq x$.

Proof. If $y \in C^{\perp}$ then the representability of $G$ ensures the existence of some normal prime subgroup $P$ omitting $y$ and therefore containing $C$. But since $C^{\perp \perp}$ is order closed, $x \in C^{\perp \perp}$ and $x \wedge y=1$ so $x \in P$. In this case $P c=P x=P$ for any $c \in C$. If $y \notin C^{\perp}$ one may assume $y \in C$; otherwise replace $y$ with $y \wedge c$ for some $c \in C$ such that $y \wedge c>1$. The fact that $x y \in \operatorname{ocl}(C)$ and $x y>x$ implies that there is some $c \in C$ with $c \leqq x y$ but $c \not \equiv x$, for otherwise $x y \neq \mathrm{V}\{c \wedge x y \mid c \in C\}$. The representability of $G$ yields a normal prime $P$ omitting $y$ such that $P c>P x$. Therefore $P(c \wedge x)=P x$.

COROLlaRy 1.23. If $C$ is a convex $\ell$-subgroup of the representable $\ell$-group $G$, and if $x_{1}, x_{2}, \cdots, x_{n} \in \operatorname{ocl}(C)$, then there is a convex Hausdorff $\ell$-topology $\mathscr{B}$ such that each $x_{i}$ is in $\operatorname{cl}(C)$.

Proof. Let $x=\left|x_{1}\right| \vee\left|x_{2}\right| \vee \cdots \vee\left|x_{n}\right| \in$ ocl $(C)$. Let $T=\left\{x c^{-1} \mid c \leqq\right.$ $x, c \in C\}$. $T$ strongly converges to 1 by the previous lemma.

COROLLARY 1.24. A convex $\ell$-subgroup of the representable $\ell-$ group $G$ is order closed if and only if it is closed with respect to every convex Hausdorff $\ell$-topology.

2. Prime topologies. The central role of prime subgroups in the theory of lattice ordered groups makes the subject of the $\ell$-topologies generated by primes unavoidable. In particular, the topologies of [15] and [6] are of these types. For $P$ a prime subgroup of the $\ell$-group $G$ let us call a set of the form $\{g \in G \mid P a<$ $P g<P b\}$ a $P$-interval and write it $(P a, P b)$. Such a set is the inverse image under the natural map of an interval of the chain of cosets of $P$.

Proposition 2.1. A prime subgroup $P$ of the t/-group $G$ is topologically closed if and only if every $P$ interval is an open set.

Proof. Suppose $P$ is a topologically closed prime subgroup of $G$. Then $\{x \mid P x \geqq P b\}$ is also closed, being the inverse image of the closed set $P b$ under the continuous map $x \rightarrow x \wedge b$. Likewise $\{x \mid P x \leqq P a\}$ is closed. Therefore $(P a, P b)$ is open. Conversely, if every $P$-interval is open then for any $1<x \notin P$ the open set $\left(P, P x^{2}\right)$ contains $x$ and is disjoint from $P$. Hence $P$ is topologically closed. 
We shall most often use the preceding proposition in the following form: a prime subgroup $P$ of the $t \ell$-group $(G, \mathscr{B})$ is topologically closed if and only if $\left(P a^{-1}, P a\right) \in \mathscr{B}$ for all $a \in G^{+}-P$. The next result is due to Madell ([16], Proposition 7).

COROLlaRY 2.2. Any prime properly containing a topologically closed prime subgroup is itself both closed and open.

Proof. If $Q$ is a convex $\ell$-subgroup properly containing the closed prime $P$, then $Q=\cup\left\{\left(P q^{-1}, P q\right) \mid q \in Q^{+}-P\right\}$. Therefore $Q$, along with each coset, is open and hence closed.

Proposition 2.3. Suppose $G$ is an $\ell$-subgroup of the t/-group $H$. The topologically closed primes of $\mathrm{cl}(G)$ are in one-to-one order preserving correspondence with those of $G$ by means of the intersection map. The inverse of this map is $P \rightarrow \operatorname{cl}(P)$.

Proof. $\mathrm{cl}(P)$ is a topologically closed prime subgroup of $\mathrm{cl}(G)$ whenever $P$ is a prime subgroup of $G$. If $P$ is closed, then $\operatorname{cl}(P) \cap$ $G=P$. Suppose $Q$ is a topologically closed prime subgroup of $\operatorname{cl}(G)$. Then $Q \cap G$ is a topologically closed prime subgroup of $G$ such that $\operatorname{cl}(Q \cap G) \subseteq Q$. Equality must hold, since otherwise $Q-$ $\operatorname{cl}(Q \cap G)$ would be a nonempty open subset of $\mathrm{cl}(G)$ which would have to intersect $G$. Therefore $\operatorname{cl}(Q \cap G)=Q$.

Proposition 2.3 should not be construed as saying that the topologically closed primes of $\mathrm{cl}(G)$ intersect to 1 just because those of $G$ do. For example, take $G$ to be the $\ell$-group of continuous real-valued functions on the real numbers $\boldsymbol{R}$ having bounded support. Let $\mathscr{B}_{1}$ be the Hausdorff $\ell$-topology generated by sets $B_{n}=\left\{f \in G\left|\int_{-\infty}^{\infty}\right| f \mid d r<1 / n\right\}$ for positive integers $n$. Observe that every prime subgroup of $G$ is contained in some maximal prime subgroup $G_{r}=\{g \in G \mid(r) g=0\}$, and that $\operatorname{cl}\left(G_{r}\right)=G$ with respect to $\mathscr{B}_{1}$. Let $\mathscr{B}_{2}$ be generated by finite intersections of the prime subgroups $G_{r}, r \in R$. Let $H$ be the cardinal product $G \oplus G$ and let $\mathscr{B}$ be the product of topologies $\mathscr{B}_{1}$ and $\mathscr{B}_{2}$. Think of $G \ell$-embedded in $H$ as the diagonal $\{(g, g) \mid g \in G\}$. It is not difficult to show that $G$ is dense in $H$; however, the intersection of the topologically closed primes of $H$ is $G \oplus 1$, while in $G$ this intersection is 1 .

If $\Gamma$ is a set of prime subgroups of an $\ell$-group $G$, we shall write $\mathscr{C}(\Gamma)$ to mean the filter generated by finite intersections of the primes from $\Gamma$ and write $\mathscr{B}(\Gamma)$ to mean the filter generated by finite intersections of the $P$-intervals $\left(P a^{-1}, P a\right), P \in \Gamma, a \in G^{+}-P$. 
The reader should realize that $\mathscr{B}(\Gamma)$ and $\mathscr{C}(\Gamma)$ may coincide.

Lemma 2.4. $\mathscr{C}(\Gamma)$ or $\mathscr{B}(\Gamma)$ is an l-topology if and only if it is a normal filter. Either topology is Hausdorff if and only if $\cap \Gamma=1$.

Proof. We prove parts (b) and (c) of Theorem 1.1 for $\mathscr{B}(\Gamma)$.

(b) Given $P \in \Gamma$ and $a \in G^{+}-P$, let $B=\left(P a^{-1}, P a\right)$ and consider $A=\left(\left(a^{-1} P a\right) a^{-1},\left(a^{-1} P a\right) a\right) \cap\left(\left(a P a^{-1}\right) a^{-1},\left(a P a^{-1}\right) a\right) \in \mathscr{B}(\Gamma)$. Then $x \in A$ implies $\left(a^{-1} P a\right) a^{-1}<\left(a^{-1} P a\right) x$ or $P x^{-1}<P a$. Similarly $P a^{-1}<P x^{-1}$. That is, $x^{-1} \in B$ so $A \cong B^{-1}$.

(c) Given $P \in \Gamma$ and $a \in G^{+}-P$, let $B=\left(P a^{-1}, P a\right)$. We seek $A \in \mathscr{B}(\Gamma)$ such that $A \cdot A \subseteq B$. If $B=P$ then let $A=P$. If not, there must be some $b$ between 1 and $a$ with $P<P b<P a$. In fact, one may assume that $P a^{-1}<P b^{-1}<P<P b<P a$. Define

$$
\begin{aligned}
& A_{1}=\left(P b^{-1}, P b\right), A_{2}=\left(\left(b^{-1} P b\right) a^{-1} b,\left(b^{-1} P b\right) b^{-1} a\right), \\
& A_{3}=\left(\left(b P b^{-1}\right) b a^{-1},\left(b P b^{-1}\right) a b^{-1}\right),
\end{aligned}
$$

and $A=A_{1} \cap A_{2} \cap A_{3} \in \mathscr{B}(\Gamma)$. If $x$ and $y$ are in $A$, then $P x y<P b y$ since $x \in A_{1}$. But in this case $P b y<P a$ since $\left(b^{-1} P b\right) y<\left(b^{-1} P b\right) b^{-1} a$ because $y \in A_{2}$. Therefore $P x y<P a$. Similarly $P x y>P a^{-1}$. Therefore $A \cdot A \subseteq B$.

Whenever they are normal filters, $\mathscr{B}(\Gamma)$ and $\mathscr{C}(\Gamma)$ will be termed the coarse and fine prime topologies of $\Gamma$, respectively. $\mathscr{C}(\Gamma)$ is a normal filter if and only if $\{P$ a prime $\mid P \supseteqq Q \in \Gamma\}$ is a normal set of primes. $\mathscr{B}(\Gamma)$ is a normal filter if and only if for every $P \in \Gamma, g \in G$, and $a \in G^{+}-g^{-1} P g$ there are primes $P_{1}, P_{2}, \cdots, P_{n}$ in $\Gamma$ and elements $a_{i} \in G^{+}-P_{i}$ such that $\cap\left(P_{i} a_{i}^{-1}, P_{i} a_{i}\right) \subseteq\left(\left(g^{-1} P g\right) a^{-1}\right.$, $\left.\left(g^{-1} P g\right) a\right)$. An important special case of the latter condition is the following. Let $G$ be a transitive $\ell$-group of order preserving permutations of the chain $\Omega$ and let $\Delta$ be a subset of $\Omega$ dense in its interval topology. If $\Gamma$ is the set of stabilizers $\left\{G_{\delta} \mid \delta \in \Delta\right\}$, then $\mathscr{B}(\Gamma)$ is a normal filter. This special case is sufficiently important to merit the terminology coarse and fine stabilizer topologies for $\mathscr{B}(\Gamma)$ and $\mathscr{C}(\Gamma)$ respectively, where $\Gamma=\left\{G_{\gamma} \mid \gamma \in \Omega\right\}$. Unless otherwise specified, we shall always assume that $\ell$-permutation groups are topologized by their coarse stabilizer topologies.

Suppose $\Gamma$ is a normal set of prime subgroups of the $\ell$-group $G$. Let $\Gamma^{\prime}\left(\Gamma^{\prime \prime}\right)$ be $\{P$ a prime $\mid P$ is closed with respect to $\mathscr{B}(\Gamma)(\mathscr{C}(\Gamma))\}$ and let $\Gamma_{r}=\{Q$ a regular prime $Q \supseteq P \in \Gamma\}$. It is not difficult to establish that $\mathscr{B}(\Gamma)=\mathscr{B}\left(\Gamma^{\prime}\right)=\mathscr{B}\left(\Gamma_{r}\right)$, that $\mathscr{C}\left(\Gamma_{r}\right) \subseteq$ $\mathscr{C}(\Gamma) \subseteq \mathscr{C}\left(\Gamma^{\prime \prime}\right)$, and that $\Gamma^{\prime}$ and $\Gamma^{\prime \prime}$ are closed with respect to 
intersections of chains. With a little more effort one may also show that each of $\mathscr{B}(\Gamma)$ and $\mathscr{C}(\Gamma)$ is order closed if and only if each $P \in \Gamma$ is order closed.

Let $\Gamma$ be the set of order closed prime subgroups of the $\ell-$ group $G$. The $\ell$-topology $\mathscr{B}(\Gamma)$ is called the $\alpha$-topology on $G$. The $\alpha$-topology is Hausdorff if and only if $G$ is completely distributive; that is, when $\cap \Gamma=1$. It was Madell [15] who first delineated the attributes of this important convex order closed $\ell$-topology. The next proposition is Theorem 4 of [15].

Proposition 2.5. The $\alpha$-topology is coarser than every Hausdorff <-topology. In particular, any completely distributive <-group has a unique coarsest Hausdorff $\ell$-topology.

Proof. If $P$ is an order closed prime and $\mathscr{B}$ is a Hausdorff $\measuredangle$-topology then $P$ is topologically closed. Therefore $\left(P a^{-1}, P a\right) \in \mathscr{B}$ for all $a \in G^{+}-P$. That is, $\mathscr{B}(\Gamma) \subseteq \mathscr{B}$, where $\Gamma$ is the set of order closed primes.

If $\left\{G_{\lambda} \mid \lambda \in \Lambda\right\}$ is a set of $\ell$-groups, let $\Pi G_{\lambda}=\left\{f: \Lambda \rightarrow \cup G_{\lambda} \mid(\lambda) f \in\right.$ $G_{\lambda}$ all $\left.\lambda \in \Lambda\right\}$ with componentwise operations and let $\Sigma G_{\lambda}=\{f \in$ $\Pi G_{\lambda} \mid(\lambda) f=1$ except for finitely many $\left.\lambda\right\}$.

Proposition 2.6. Suppose $\left\{G_{\lambda} \mid \lambda \in \Lambda\right\}$ is a set of t-groups. The $\alpha$-topology on $\Pi G_{\lambda}$ is the product of the $\alpha$-topologies on each $G_{\lambda}$, and the $\alpha$-topology on $\Sigma G_{\lambda}$ is inherited from the $\alpha$-topology on $\Pi G_{\lambda}$.

Proof. The proof is an immediate consequence of the fact that the order closed primes of $\Pi G_{\lambda}$ and $\Sigma G_{\lambda}$ are precisely those of the form $\left(P_{\lambda}\right) \pi_{\lambda}^{-1}$, where $P_{\lambda}$ is an order closed prime of $G_{\lambda}$ and $\pi_{\lambda}$ is the projection map from the product onto $G_{\lambda}$.

The importance of the $\alpha$-topology derives in part from the importance of completely distributive $\ell$-groups. For example, the targets of three major representation theorems, namely $A(S), V$, and cardinal products of totally ordered groups, are all completely distributive. Also, the $\alpha$-topology on a totally ordered $\ell$-group is the interval topology, the only nontrivial convex Hausdorff $\ell$ topology. In an $\ell$-permutation group, the $\alpha$-topology and the coarse stabilizer topology coincide whenever stabilizers are order closed.

Two questions arise naturally from Proposition 2.5. Can a non completely distributive $\ell$-group have a coarsest Hausdorff $\ell$-topology? Is the $\alpha$-topology the finest $\ell$-topology coarser than every Hausdorff $\measuredangle$-topology? It is possible to decide these matters in the represent- 
able case. We remind the reader that every group topology is regular; that is, every neighborhood of $g \in G$ contains a closed neighborhood of $g$.

THeORem 2.7. Suppose $G$ is a representable l-group and that $\Gamma$ is the set of order closed primes of $G$. Then any <-topology $\mathscr{D}$ coarser than every Hausdorff $\ell$-topology must be coarser than $\mathscr{C}(\Gamma)$.

Proof. Consider an arbitrary neighborhood $D \in \mathscr{D}$. We may assume $D$ to be closed with respect to $\mathscr{D}$. Let $\Delta$ be the set of all prime subgroups of $G$. By assumption, $\mathscr{D}$ is coarser than $\mathscr{C}(\Delta)$ so that there must be primes $P_{1}, P_{2}, \cdots, P_{n}$ with $D \supseteqq \cap P_{i}$. Let $X$ be the closure of $\cap P_{i}$ with respect to $\mathscr{D}$. By Propositions 1.8 and 1.23, $X$ is an order closed convex $\ell$-subgroup contained in $D$. But this implies that $\cap \operatorname{ocl}\left(P_{i}\right)=\operatorname{ocl}\left(\cap P_{i}\right) \subseteq X \subseteq D$. The upshot of all this is that $D \in \mathscr{C}(\Gamma)$.

COROLLARY 2.8. A representable l-group has a coarsest Hausdorff <-topology if and only if it is completely distributive.

In some cases Theorem 2.7 may be the best result one can expect. If $\Gamma$ is the set of order closed prime subgroups of $G$, then $\cap \Gamma$ is called the distributive radical of $G$, written $D(G)$. The lex product of an $\ell$-group $G$ with the real numbers $\boldsymbol{R}$, written $G \overleftarrow{\times} \boldsymbol{R}$, is the group product $G \times \boldsymbol{R}$ ordered by $\left(g_{1}, n\right) \leqq\left(g_{2}, m\right)$ if $n<m$ or $n=m$ and $g_{1} \leqq g_{2}$. Notice that the only proper order closed prime subgroups of $G \overleftarrow{\times} \boldsymbol{R}$ are those of the form $P \times 0$, where $P$ is an order closed prime subgroup of $G$.

Proposition 2.9. Suppose $G$ is an $\ell$-group such that $D(G)=G$, that $H$ is $G \overleftarrow{\times} \boldsymbol{R}$, and that $\Gamma$ is $\{G \times 0\}$. Then, on $H, \mathscr{C}(\Gamma)$ is strictly finer than the $\alpha$-topology $\mathscr{B}(\Gamma)$, and $\mathscr{C}(\Gamma)$ is the finest $\iota$-topology coarser than every Hausdorff $\ell$-topology.

Proof. Consider an arbitrary Hausdorff $\ell$-topology $\mathscr{B}$ on $H$. Find disjoint $g_{1}, g_{2} \in G$ with neither $g_{1}$ nor $g_{2}$ the identity. Since $\mathscr{B}$ is Hausdorff there must be some $B \in \mathscr{B}$ containing neither $h_{1}=$ $\left(g_{1}, 0\right)$ nor $h_{2}=\left(g_{2}, 0\right)$. Since $h_{1} \wedge h_{2}=1$ there must be an $A \in \mathscr{B}$ such that $A h_{1} \wedge A h_{2} \subseteq B$ and $A=A^{-1}$. This forces $A \subseteq G \times 0$, since $(g, r) \in A$ with $r>0$ implies $h_{1}, h_{2} \in B$. That is, $G \times 0 \in \mathscr{B}$ so $\mathscr{B}$ is finer than $\mathscr{C}(\Gamma)$.

Suppose $T$ is a filter on $G^{+}$with $\Lambda T=1$ and that $P$ is an 
order closed prime subgroup. Since the natural map preserves infima, $P=\Lambda\{P t \mid t \in T\}$. This observation shows that the $\alpha$-topology on any $\ell$-group is $T$-coarse for every filter $T$ such that $\Lambda T=1$.

Proposition 2.10. Any sublattice of an 6 -group which is closed with respect to the $\alpha$-topology is order closed. A convex sublattice of a completely distributive $\measuredangle$-group is order closed if and only if it is closed with respect to the $\alpha$-topology.

It is interesting to observe that the representable and completely distributive cases of a result of Byrd and Lloyd [4] may be proved by topological means.

Proposition 2.11. In a representable or completely distributive $\measuredangle$-group $G$, any convex $\ell$-subgroup containing an order closed prime subgroup is itself order closed and prime.

Proof. Suppose $Q$ is a convex $\ell$-subgroup properly containing the order closed prime subgroup $P$ of $G$. By Theorem $1.14 P$ is closed with respect to any Hausdorff $\ell$-topology. By Corollary 2.2, $\mathrm{Q}$ is also closed with respect to every Hausdorff $\ell$-topology. By either Corollary 1.23 or Proposition $2.10 Q$ is order closed.

We close this section by remarking that results 1.18 and 1.20 can also be proven by producing Hausdorff prime topologies with the desired properties. This seems to indicate that there is a plenitude of such topologies in existence.

3. Semantic links between $G$ and $\mathrm{cl}(G)$. Suppose $G$ is an $\ell$-subgroup of the $t \ell$-group $(H, \mathscr{B})$. A strong semantic link exists between $G$ and $\mathrm{cl}(G)$. Let $c_{1}, c_{2}, \cdots, c_{m}$ be constants from $H$, and let $W_{1}, W_{2}, \cdots, W_{n}$ be words built up from the $c_{i}$ 's and from variables $v_{1}, v_{2}, \cdots, v_{k}$ using group and lattice operations. Let us agree to term any formula obtained by joining the atomic formulas " $W_{i}=$ 1 " by conjunction and disjunction a disjunctive formula. If $\psi$ is a formula having free variables $v_{1}, v_{2}, \cdots, v_{k}$ we shall abbreviate its universal closure $\forall v_{1}, v_{2}, \cdots, v_{k} \psi$ by $\forall v \psi$. Finally, $\langle X\rangle$ is the $\ell$-subgroup of $H$ generated by a subset $X$ of $H$.

THEOREM 3.1. Suppose $G$ is an l-subgroup of the Hausdorff t/-group $H$. Let $\psi$ be any disjunctive formula mentioning constants $c_{1}, c_{2}, \cdots, c_{m}$ in $H$. Then $\left\langle G, c_{1}, c_{2}, \cdots, c_{m}\right\rangle \vDash \forall v \psi$ if and only if $\left\langle\operatorname{cl}(G), c_{1}, c_{2}, \cdots, c_{m}\right\rangle \vDash \forall v \psi$.

Proof. Since implication from right to left is clear, suppose 
$\left\langle\operatorname{cl}(G), c_{1}, c_{2}, \cdots, c_{m}\right\rangle \vDash \forall v \psi$. We may assume $\psi$ is of the form " $W_{1}=1$ or $W_{2}=1$ or $\cdots$ or $W_{n}=1$." Thus we are assuming we have elements $h_{i} \in\left\langle\operatorname{cl}(G), c_{1}, c_{2}, \cdots, c_{m}\right\rangle$ such that $W_{j}\left(h_{1}, h_{2}, \cdots, h_{k}\right) \neq 1$ for $1 \leqq j \leqq n$. The continuity of the operations now assures the existence for each $i$ of a neighborhood $J_{i}$ of $h_{i}$ such that $1 \notin W_{j}\left(J_{1}\right.$, $J_{2}, \cdots, J_{k}$ ) for $1 \leqq j \leqq n$. The continuity of the operations also implies that $\left\langle G, c_{1}, c_{2}, \cdots, c_{m}\right\rangle$ is topologically dense in $\left\langle\mathrm{cl}(G), c_{1}\right.$, $\left.c_{2}, \cdots, c_{m}\right\rangle$. Therefore, choosing $g_{i} \in G \cap J_{i}$, one obtains $1 \neq W_{j}\left(g_{1}\right.$, $\left.g_{2}, \cdots, g_{k}\right)$ for $1 \leqq j \leqq n$. That is, $\left\langle G, c_{1}, c_{2}, \cdots, c_{m}\right\rangle \vDash \forall v \psi$.

COROLLARY 3.2. If $G$ is any $\ell$-subgroup of the Hausdorff t $t$ group $H$, then $G$ and $\mathrm{cl}(G)$ generate the same variety of $\ell$-groups. Moreover, $G$ is totally ordered if and only if $\mathrm{cl}(G)$ is.

Proof. $G$ is totally ordered if and only if $G \models \forall x, y\left((x \vee y) x^{-1}=\right.$ 1 or $\left.(x \vee y) y^{-1}=1\right)$.

Theorem 3.1 provides a simple proof of the representable and completely distributive cases of a (generalization of a) result due to Bernau [3].

Proposition 3.3. If $G$ is an (convex) $\ell$-subgroup of the (representable or) completely distributive $<$-group $H$, then $G$ and ocl $(G)$ satisfy the same disjunctive formulas and therefore generate the same varieties of $\ell$-groups.

Proof. Suppose ocl $(G) \vDash \psi\left(h_{1}, h_{2}, \cdots, h_{k}\right)$ for the disjunctive formula $\psi$ and $h_{1}, h_{2}, \cdots, h_{k} \in \operatorname{ocl}(G)$. By results 1.23 and 2.10 there is a Hausdorff $\ell$-topology on $H$ with respect to which $h_{1}, h_{2}, \cdots, h_{k} \in$ $\operatorname{cl}(G)$. Theorem 3.1 now implies $G \vDash \forall v \psi$.

The centralizer of a subset $X$ of an $\ell$-group $G$ is the $\ell$-subgroup $\left\{g \in G \mid g x g^{-1} x^{-1}=1, x \in X\right\}$. It follows from the next result that centralizers are closed with respect to any Hausdorff $\ell$-topology and therefore, in completely distributive $t \ell$-groups at least, order closed. In particular, if $G$ is a periodic $\ell$-subgroup of $A(S)$ (defined in [9]) and if $A(S)$ is endowed with any Hausdorff $\iota$-topology then cl $(G)$ and ocl $(G)$ are periodic with the same period as $G$.

Corollary 3.4. If $C$ is maximal among those (convex) $\ell$-subgroups of the (representable or) completely distributive ८-group $H$ which satisfy a fixed set of disjunctive formulas then $C$ is order closed. 
Just as the rationals may be completed by Dedekind cuts to yield the real numbers, so an arbitrary $\ell$-group may be completed by cuts to yield its Dedekind-MacNeille completion ([8]). For $X$ a subset of the lattice $G$ let $L(X)=\{g \in G \mid g \leqq X\}$ and $U(X)=\{g \in$ $G \mid g \geqq X\}$. A subset $X$ of a lattice $G$ is a cut if $\phi \neq X \neq G$ and $L U(X)=X$. Notice that $L(g)$ is a cut for each $g \in G$. For cuts $X_{1}$ and $X_{2}$ we define $X_{1} \leqq X_{2}$ if $X_{1} \leqq X_{2}$ and $X_{1} \cdot X_{2}=L U\left(X_{1} X_{2}\right)$. These conventions make the set of cuts of a lattice into a lattice and the set of cuts of an $\ell$-group into a partially ordered semigroup with identity $L(1) . G^{D}$ designates the $\ell$-group of units of this semigroup; specifically, $G^{D}=\left\{X \mid X\right.$ is a cut and $\left.X \cdot L\left(X^{-1}\right)=L(1)\right\}$. It is light work to prove that $G^{D}$ is an $\ell$-group in which $G$ is $\ell$-embedded via $g \rightarrow L(g)$. One may also show ([1]) that for any $\measuredangle$-group $H$ having $G$ as an $\ell$-subgroup there is an $\ell$-monomorphism $\theta: H \rightarrow G^{D}$ with $\theta$ the identity on $G$ if and only if every $h \in H^{+}$ satisfies $h=\mathbf{V}(L(h) \cap G)=\Lambda(U(h) \cap G)$.

CoRollary 3.5. If $G$ is a completely distributive $\ell$-group then $G$ and $G^{D}$ satisfy the same disjunctive formulas.

Proof. It is clear that ocl $(G)$ in $G^{D}$ is $G^{D}$.

The following corollary is part of a result due to Bernau ([3]).

COROLlaRY 3.6. If $G$ is a completely distributive l-group then $G$ and its lateral completion $G^{L}$ satisfy the same disjunctive formulas.

Proof. It is clear that $\operatorname{ocl}(G)$ in $G^{L}$ is $G^{L}$.

4. Continuous representation theorems. When can a representation of the $\ell$-subgroup $G$ of the $t /$-group $H$ be extended to a continuous representation of $\mathrm{cl}(G)$ ? The next result provides a necessary condition for such a representation since the targets of the classical representation theorems are completely distributive.

Proposition 4.1. Suppose $\theta$ is an $\ell$-monomorphism from the

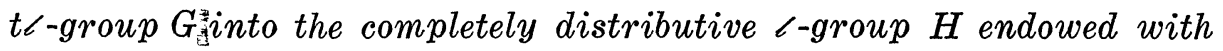
some Hausdorff $\ell$-topology. If $\theta$ is continuous then the topologically closed prime subgroups of $G$ must intersect to 1.

Proof. Under the circumstances $\theta$ must be continuous with respect to the $\alpha$-topology on $H$. Hence $(P) \theta^{-1}$ is a topologically closed prime subgroup of $G$ for each order closed prime subgroup 
$P$ of $H$.

It is necessary to introduce some ideas from $\ell$-permutation group theory. These concepts are due to Holland in the main [13], though the most comprehensive exposition and the terminology may be found in [9]. Suppose $G$ is a transitive $\ell$-group of order preserving permutations of the chain $\Omega$. Let $\Delta$ be the set of convex $G$-congruences on $\Omega$ other than the identity congruence. Let $\bar{\Omega}$ be the chain obtained by completing $\Omega$ by Dedekind cuts. Let $\Omega^{0}$ be $\left\{\gamma \in \bar{\Omega} \mid\right.$ for every $\mathscr{C} \in \Delta$ there are $\gamma_{1}, \gamma_{2} \in \Omega$ with $\gamma_{1} \mathscr{C} \gamma_{2}$ and $\left.\gamma_{1}<\gamma<\gamma_{2}\right\}$. Glass gives other definitions of $\Omega^{0}$ on pages 247 and 278 of [9]. The reader should realize that $\Omega \subseteq \Omega^{0} \subseteq \bar{\Omega}$ and that each $g \in G$, considered to be permuting $\Omega$, actually maps $\Omega^{0}$ onto $\Omega^{0}$. For each $\mathscr{C} \in \Delta$ let $\mathscr{C}^{\sim}$ be the equivalence relation on $\Omega^{0}$ obtained by convexifying the $\mathscr{C}$ classes. Finally, let $G^{0}=\left\{h \in A\left(\Omega^{\circ}\right) \mid h\right.$ respects $\mathscr{C}^{\sim}$ for each $\left.\mathscr{C} \in \Delta\right\}$. It should be clear that $G \leqq G^{r}$ and that the convex $G^{0}$ congruences on $\Omega^{0}$ are precisely those of the form $\mathscr{C}^{-}$for $\mathscr{C} \in \Delta$. Put another way, intersection provides a one-to-one order preserving correspondence between the convex $\ell$ subgroups of $G^{0}$ containing the stabilizer $G_{\gamma}^{0}$ and those of $G$ containing $G_{\gamma}$ for each $\gamma \in \Omega$.

One might naturally wonder whether $G^{0}$ is transitive on $\Omega^{0}$. The answer to this query must be negative in general; for example, the $\ell$-permutation group $G$ constructed in [13] is doubly transitive on its chain $\Omega$, so that $\Omega^{0}=\bar{\Omega}$. Nevertheless, $G^{0}=A(\bar{\Omega})$ fails to be transitive on $\bar{\Omega}$. However, if $G$ has no finest nonidentity convex $G$ congruence then $G^{0}$ must be transitive on $\Omega^{0}$.

Proposition 4.2. If $G$ is a transitive but not locally o-primitive $\ell$-group of order preserving permutations of the chain $\Omega$, then $G^{0}$ is transitive on $\Omega^{0}$.

Proof. It is sufficient to find, for $\gamma \in \Omega$ and $\delta \in \Omega^{0}$, some $h \in G$ such that $(\gamma) h=\delta$. Let $\left\{\mathscr{C}_{\alpha} \mid \alpha<\beta\right\}$ be a subscripting of certain of the members of $\Delta$ with the following properties.

(a) $\mathscr{C}_{0}$ is the large congruence. That is, $\gamma_{1} \mathscr{C}_{0} \gamma_{2}$ for all $\gamma_{1}, \gamma_{2} \in \Omega$.

(b) $\mathscr{C}_{\alpha+1}$ is strictly finer than $\mathscr{C}_{\alpha}$.

(c) For limit ordinals $\eta, \mathscr{C}_{\eta}$ is the coarsest congruence finer than each $\mathscr{C}_{\alpha}, \alpha<\eta$.

(d) For every $\mathscr{C} \in \Delta$ there is some $\alpha<\beta$ with $\mathscr{C}_{\alpha}$ finer than $\mathscr{C}$.

For each $\alpha<\beta$ let $g_{\alpha} \in G$ satisfy $(\gamma) g_{\alpha} \mathscr{C}_{\alpha+1} \delta$. Requirements (a) $\sim(\mathrm{d})$ guarantee that for each $\rho \in \Omega^{0}, \rho \neq \gamma$, there is a unique $\alpha<\beta$ such that $\rho \mathscr{C}_{\alpha} \sim \gamma$ but $\rho \mathscr{C}_{\alpha+1} \gamma$. Define $h: \Omega^{0} \rightarrow \Omega^{0}$ by 


$$
(\rho) h=\left\{\begin{array}{l}
\delta \text { for } \rho=\delta \\
(\rho) g_{\alpha} \text { where } \rho \mathscr{C}_{\alpha} \gamma \gamma \text { but } \rho \mathscr{C}_{\alpha+1} \gamma .
\end{array}\right.
$$

The reader may check that $h \in G^{0}$. It is amusing to notice that $(\Omega) h=\Omega \cup\{\delta\}$.

There is a connection between $G^{D}$ and $\Omega^{0}$ when $G$ is a uniquely transitive $\iota$-group of order preserving permutations of the chain $\Omega$. In this case one may take $\Omega$ to be $G$ itself. But the cuts added to $G$ to form the totally ordered group $G^{D}$ are precisely those cuts added to $\Omega$ to form $\Omega^{0}$.

Proposition 4.3. Suppose $G$ is a uniquely transitive $\ell$-group of order preserving permutations of the chain $\Omega$. Then $\mathrm{cl}(G)$ with respect to the coarse stabilizer topology on $G^{0}$ is transitive on $\Omega^{0}$ and is $\ell$-isomorphic to $G^{D}$.

Proof. Suppose $\theta$ is the canonical order isomorphism from $G$ onto $\Omega$. By the remark preceding the proposition $\theta$ may be uniquely extended to an order isomorphism from $G^{D}$ onto $\Omega^{0}$. $\theta$ then induces an $\ell$-isomorphism $\hat{\theta}$ from $G^{D}$ into $G^{0}$ in the following fashion. For each $t \in G^{D}$ define $(t) \hat{\theta}=g_{t} \in G^{0}$ by $(\delta) g_{t}=(\delta) \theta^{-1} t \theta$ for all $\delta \in \Omega^{0}$. Since $\left(G^{D}\right) \hat{\theta}$ is clearly transitive on $\Omega^{r}$ it follows that $\left(G^{D}\right) \hat{\theta}$ is maximal among totally ordered $\ell$-subgroups of $G^{0}$. On the other hand $G$ is clearly dense in $\left(G^{D}\right) \hat{\theta}$ with respect to the coarse estabilizer topology on $G^{0}$. Since $\mathrm{cl}(G)$ must be totally ordered, it follows that $\mathrm{cl}(G)=$ $\left(G^{D}\right) \hat{\theta}$.

Theorem 4.5 is a useful description of those $\ell$-subgroups of a transitive $\ell$-permutation group dense in its coarse stabilizer topology. The next result lays the foundation for this description. The notation $G \leqq K$ means that $G$ is an $\ell$-subgroup of $K$.

LemMA 4.4. Suppose $G$ is an $\ell$-subgroup of the transitive $\ell$-group $K$ of order preserving permutations of the chain 1 . Then the following conditions (a) and (b) together are equivalent to condition (c).

(a) For any $\delta \in \Lambda$ the orbit $(\delta) G$ is dense in the interval topology on $\Lambda$.

(b) For any $\delta \in \Lambda$ and any convex $G$ congruence $\mathscr{C}$ on $(\delta) G$, the equivalence relation $\mathscr{C}^{\sim}$ obtained by convexifying the classes of $\mathscr{C}$ in $\Lambda$ is a convex $K$ congruence on $\Lambda$.

(c) For any $\delta \in \Lambda, G$ acts faithfully on $\Omega=(\delta) G, \Lambda \cong \Omega^{0}$, and $K \leqq G^{0}$. 
Proof. If (c) holds then (a) follows from $\Lambda \subseteq \Omega^{0}$ and (b) follows from $K \leqq G^{n}$. Now suppose that (a) and (b) hold, $\delta \in \Lambda$ is fixed, and that $\Omega=(\delta) G$. It follows from (a) that $G$ acts faithfully on $\Omega$ and that $\Lambda \leqq \bar{\Omega}$. In order to show that $\Lambda \subseteq \Omega^{0}$ consider $\gamma \in \Lambda$ and $\mathscr{C}$ a nonidentity convex $G$ congruence on $\Omega$. By (b) $\mathscr{C}^{\sim}$ is a nonidentity convex $K$ congruence on $\Lambda$ so that $\gamma \mathscr{C}^{\sim}$ is a nonempty interval of $\Lambda$ without first or last element. By (a) there must be elements $\gamma_{1}, \gamma_{1} \in \Omega$ with $\gamma_{1} \mathscr{C} \gamma_{2}$ and $\gamma_{1}<\gamma<\gamma_{2}$. Since $\mathscr{C}$ was arbitrary, $\gamma \in \Omega^{0}$. Finally, each permutation $k \in K$ must respect each $\mathscr{C}^{\sim}$ for $\mathscr{C}$ a convex $G$ congruence on $\Omega$ because of (b). That is, $K \leqq G^{0}$.

Theorem 4.5. Suppose $K$ is a transitive 6 -group of order preserving permutations of the chain $\Lambda$. If $G$ is an $\ell-s u b g r o u p$ dense in the coarse stabilizer topology on $K$, then for any $\delta \in \Lambda G$ acts faithfully on $\Omega=(\delta) G, \Lambda \leqq \Omega^{0}$, and $K \leqq G^{0}$.

Proof. We need only verify conditions (a) and (b) of Lemma 4.4. To check condition (a), fix $\delta \in \Lambda$ and consider $\eta, \rho \in \Lambda$ with $\eta<\rho$. We must produce an element $g \in G$ such that $\eta \leqq(\delta) g<\rho$. It may happen that no member of $\Lambda$ lies between $\eta$ and $\rho$. In this case the stabilizer $K_{\eta}$ is $\left(K_{\eta} k^{-1}, K_{\eta} k\right)$ for any $k \in K^{+}$such that $(\eta) k=\rho$. Therefore $K_{\eta}$ is an open set. Since $\{k \in K \mid(\delta) k=\eta\}$ can be written $k_{0} K_{\eta}$ for any particular $k_{0} \in K$ taking $\delta$ to $\eta$, it follows that this set is open also. Therefore there must be some $g \in G$ taking $\delta$ to $\eta$. In case $\eta<\gamma<\rho$ for some $\gamma \in \Lambda$, choose $k \in K^{+}-K_{r}$ such that $\eta \leqq(\gamma) k^{-1}<\gamma<(\gamma) k \leqq \rho$ and choose $m \in K$ such that $(\delta) m=\gamma$. Then there must be some $g \in G \cap m\left(K_{\gamma} k^{-1}, K_{r} k\right)$. For such a $g, \eta<(\delta) g<\rho$.

It remains to check (b) of Lemma 4.4. To that end fix $\delta \in \Lambda$, let $\Omega=(\delta) G$ and consider a nonidentity convex $G$ congruence $\mathscr{C}$ on $\Omega$. Let $P$ be $\{g \in G \mid(\delta) g \mathscr{C} \delta\}$, a prime subgroup of $G$ properly containing $G_{j}$. Recall that $P$ canonically induces $\mathscr{C}$ in the sense that, for $\eta, \gamma \in \Omega, \eta \mathscr{C} \gamma$ if and only if whenever $(\delta) g_{1}=\eta$ and $(\delta) g_{2}=\gamma$ it follows that $g_{1} g_{2}^{-1} \in P$. By Proposition 2.3 there is a unique prime subgroup $Q$ of $K$ properly containing $K_{\delta}$ such that $Q \cap G=P$. Q canonically induces a convex $K$ congruence $\mathscr{D}$ on $\Lambda$; that is, $\gamma \mathscr{D} \eta$ if whenever $(\delta) k_{1}=\gamma$ and $(\delta) k_{2}=\eta$ it is true that $k_{1} k_{2}^{-1} \in Q$. Clearly $\mathscr{C}$ is the restriction of $\mathscr{D}$ to $\Omega$. Finally, since each $\mathscr{D}$ class $D$ is a nonempty interval of $\Lambda$ without first or last element, $\Omega \cap D$ is coterminal in $D$. That is, $\mathscr{C}^{\sim}=\mathscr{D}$.

We propose to use products of various $G^{0}$ 's as targets in the representation theorems. The next two propositions argue in favor 
of the suitability of $G^{0}$ for this purpose. The first says that $G^{0}$ ought to be big enough, and the second says it ought to be complete enough.

Proposition 4.6. If $G$ is an $\ell$-permutation group acting transitively on the chain $\Omega$, then $G^{0}$ is a depressible and laterally complete l-subgroup of $A\left(\Omega^{0}\right) . \quad G^{0}$ is order dense in $A(\bar{\Omega})$ and is therefore completely distributive.

Proof. The definition of $G^{0}$ makes the first statement clear. Consider now an arbitrary $1<h \in A(\bar{\Omega})$ and $\gamma \in \Omega$ with $(\gamma) h>\gamma$. Suppose there is a nonidentity convex $G$ congruence $\mathscr{D}$ such that $n,(\gamma) h^{-1} \leqq \gamma \mathscr{D} \leqq(\gamma) h$. Find $1<g \in G^{0}$ which moves $\gamma$ up and has support contained in $\gamma \mathscr{D}$. Clearly $h>g>1$. If no such $\mathscr{D}$ exists then there must be a finest nonidentity convex $G$ congruence $\mathscr{C}$. Find $1<g \in G^{0}$ which moves $\gamma$ up and has support contained in $\gamma \mathscr{C}$. Then $h \geqq h \wedge g>1$ and $h \wedge g \in G^{0}$.

Proposition 4.7. Suppose $G$ is an <-permutation group acting transitively on the chain $\Omega$. Then $G^{0}$ is both order closed and closed with respect to every Hausdorff l-topology on $A\left(\Omega^{\circ}\right)$ or on $A(\bar{\Omega})$.

Proof. It is sufficient to prove $G^{0}$ closed with respect to the $\alpha$-topology on $A(\bar{\Omega})$. Fix $\delta \in \Omega$, let $K=\operatorname{cl}\left(G^{0}\right)$ in $A(\bar{\Omega})$, and let $\Lambda$ be the orbit $(\delta) K$. Then $K$ is transitive and faithful on $\Lambda$. It should be clear that the construction of $\Omega^{0}$ and $G^{0}$ from $\Omega$ and $G$ yields nothing new when iterated. Therefore, by Theorem 4.5, $K=G^{0}$.

An $\ell$-permutation group $H$ is locally doubly transitive on the chain $A$ if there is a finest nonidentity convex $H$ congruence $\mathscr{C}$ and if for all $\alpha, \beta, \gamma, \delta \in \Lambda$ with $\alpha \mathscr{C} \beta, \gamma \mathscr{C} \delta, \alpha<\beta, \gamma<\delta$, there is some $h \in H$ with $(\alpha) h=\gamma$ and $(\beta) h=\delta . \quad H$ is doubly transitive if $\mathscr{C}$ is the large trivial congruence. It is well known that an $\ell$-permutation group $H$ acting transitively on the chain $A$ is locally doubly transitive if and only if there is a finest nonidentity convex $H$ congruence $\mathscr{C}$ and if for any $\gamma, \delta \in \Lambda^{0}$ with $\gamma \mathscr{C} \sim \delta, G_{\gamma} \leqq G_{\delta}$ implies $\gamma=\delta$.

LEMMA 4.8. Suppose $H$ is an $\ell$-permutation group acting transitively on the chain 1 . Then for any $\ell$-subgroup $G$ of $H$ and any $\delta \in \bar{\Lambda}, \operatorname{cl}\left(G_{\hat{o}}\right)=\operatorname{cl}(G)_{\hat{o}}$ with respect to the coarse stabilizer topology on $H$. 
Proof. By Proposition 2.1 the stabilizers $\mathrm{cl}(G)_{\delta}$ and $G_{\delta}$ are topologically closed. Since $\mathrm{cl}(G)_{\delta} \cap G=G_{\delta}$, the lemma follows at once from Proposition 2.3.

Proposition 4.9. Suppose $H$ is an ל-permutation group acting transitively on the chain $\Lambda$ and that $G$ is an $\ell$-subgroup of $H$ whose closure in the coarse stabilizer topology is faithful on the orbit $(\delta) \operatorname{cl}(G)$ for some $\delta \in \Lambda$. Then $G$, which acts faithfully on $(\delta) G$, is locally doubly transitive on $(\delta) G$ if and only if $\mathrm{cl}(G)$ is locally doubly transitive on $(\delta) \mathrm{cl}(G)$.

Proof. Let $K$ be cl $(G)$, let $\mathscr{B}$ be the restriction to $K$ of the coarse stabilizer topology on $H$, and let $\mathscr{C}$ be the coarse stabilizer topology of $K$ thought of as permuting the orbit $(\delta) K$. Since $\mathscr{B}$ is finer than $\mathscr{C}$ and $G$ is dense in $(K, \mathscr{B}), G$ must be dense in $(K, \mathscr{C})$. Theorem 4.5 implies that $G$ acts faithfully on $\Omega=(\delta) G$, that $(\delta) K \subseteq \Omega^{0}$, and that $K \leqq G^{0}$. The proof is completed by the proof of Lemma 4.8 and by Proposition 2.3, which provide a one-toone order preserving correspondence between the stabilizers of $G$ and those of $K$.

Proposition 4.10. Suppose $H$ is an ८-permutation group acting doubly transitive on the chain $A$ and that $G$ is an l-subgroup of $H$ transitive on $\Lambda$. Then $G$ is doubly transitive on $\Lambda$ if and only if $G$ is dense in the fine stabilizer topology on $H$ if and only if $G$ is dense in the coarse stabilizer topology on $H$.

Proof. If $G$ is doubly transitive then it is $n$-fold transitive and hence clearly dense in the fine and coarse stabilizer topologies. On the other hand if $G$ is dense in the coarse stabilizer topology it must be doubly transitive by the previous result.

Let $G$ be an $\ell$-permutation group acting doubly transitively on the chain $\Omega$. By Proposition 4.10, $G$ is dense in the coarse stabilizer topology on $A(\Omega)$. In [2] is proved that any doubly transitive $A(\Omega)$ fails to be normal valued. By Proposition $3.3 G$ itself must fail to be normal valued. If we allow ourselves to use the fact that normal valued $\ell$-groups form the maximal proper variety [11], we have a proof that any doubly transitive $\ell$-permutation group $G$ generates the variety of all $\ell$-groups. This was first proved by Holland in [11].

The previous methods may be used to prove the next proposition. 
Proposition 4.11. Suppore $H$ is an $\ell$-permutation group acting transitively on the chain $A$ and that $p \in H$ has a centralizer $C$ wgich $i s$ o-primitive and periodic with period p. A transitive $\ell$-subgroup $G$ of $C$ is periodic with period $p$ if and ouly if $G$ is dense in the fine stabilizer topology on $C$ if and only if $G$ is dense in the coarse stabilizer topology on $C$.

Suppose $G$ is an $\ell$-permutation group acting transitively on the chain 1 . Let $G^{*}$ be the closure of $G$ in the coarse stabilizer topology on $G^{0}$. The condition for $h \in G^{0}$ to be in $G^{*}$ is the following. For every finite collection $\left\{\alpha_{i}, \beta_{i}, \gamma_{i} \mid 1 \leqq i \leqq n\right\}$ of points of $\Omega^{0}$ such that $\alpha_{i}<\beta_{i}<\gamma_{i}$ there must be some $g \in G$ with $\left(\alpha_{i}\right) h<\left(\beta_{i}\right) g<$ $\left(\gamma_{i}\right) h$ and $\left(\alpha_{i}\right) g<\left(\beta_{2}\right) h<\left(\gamma_{i}\right) g$ for each $i, 1 \leqq i \leqq n$. $G^{*}$ must be order closed as well as closed in every Hausdorff $\ell$-topology on $G^{0}$, $A\left(\Lambda^{0}\right)$, or $A(\bar{\Lambda})$. It follows that $G^{*}$ is laterally complete though not always depressible. The author is willing to conjecture that stabilizers are order closed in $G^{*}$. If this conjecture is true then $G^{*}$ is completely distributive and $G$ is order dense in $G^{*}$ if and only if stabilizers are order closed in $G$. (Stephen McCleary has informed the author that he has a proof of this conjecture.)

There is a close relationship between the $o$-primitive components of $G$ and those of $G^{*}$. Consider a covering pair $(\mathscr{C}, \mathscr{D})$ of convex $G$ congruences on $\Lambda$ and the corresponding covering pair $\left(\mathscr{C}^{\sim}, \mathscr{D}^{\sim}\right)$ of convex $G^{*}$ congruences on $\Lambda^{0}$. Fix $\delta \in \Lambda$ and let $\Omega\left(\Omega^{*}\right)$ be the chain $\delta \mathscr{D} / \mathscr{C}\left(\delta \mathscr{D}^{\sim} / \mathscr{C}^{\sim}\right)$ of all $\mathscr{C}$ classes ( $\mathscr{C}^{\sim}$ classes) in $\delta \mathscr{D}\left(\delta \mathscr{D}^{\sim}\right)$. The convexification map takes $\Omega$ into $\Omega^{*}$ in an order preserving way so that we may think of $\Omega$ as being a subset of $\Omega^{*}$. In fact, $\Omega$ is $\Omega^{*}$ unless $\mathscr{C}$ is the identity congruence, in which case $\Omega^{*}$ is $\bar{\Omega}$. Let $H\left(H^{*}\right)$ be the image of $\{g \in G \mid(\delta) g \mathscr{D} \delta\}\left(\left\{h \in G^{*} \mid(\delta) h \mathscr{D} \sim \delta\right\}\right)$ under the natural $\ell$-homomorphism into $A(\Omega)\left(A\left(\Omega^{*}\right)\right)$. Observe that these conventions make $H$ an $\ell$-subgroup of $H^{*} . H$ and $H^{*}$ are the $o$-primitive components of $G$ and $G^{*}$ corresponding to $(\mathscr{C}, \mathscr{D})$ and $\left(\mathscr{C}^{\sim}, \mathscr{D}^{\sim}\right)$. The point is that if $\mathscr{C}$ is the identity congruence on $\Lambda$ then $H^{*}$ is an $\ell$-subgroup of the closure of $H$ in the coarse stabilizer topology on $A\left(\Omega^{*}\right)$ and if $\mathscr{C}$ is not the identity congruence then $H^{*}$ is an $\ell$-subgroup of the closure of $H$ in the fine stabilizer topology on $A\left(\Omega^{*}\right)$. McCleary's classification theorem [17] provides a description of the possibilities for $H$ and $H^{*}$.

THEOREM 4.12. Suppose $H$ is a transitive o-primitive $\ell$-permutation group acting on the chain $\Omega$. Then one of the following occurs.

(a) $H$ is totally ordered. In this case $H$ is l-isomorphic to a subgroup of the real numbers and is closed in the fine stabilizer 
topology on $A(\bar{\Omega})$. The closure of $H$ with respect to the coarse stabilizer topology on $A(\bar{\Omega})$ is <-isomorphic to either the integers or the real numbers depending on whether $H$ has a least strictly positive element or not.

(b) $H$ is periodic with period $p \in A(\bar{\Omega})$. In this case the closure of $H$ in the fine (coarse) stabilizer topology on $A(\Omega)(A(\bar{\Omega}))$ is the centralizer of $p$ in $A(\Omega)(A(\bar{\Omega}))$. The closure of $H$ in the fine stabilizer topology on $A(\bar{\Omega})$ is $\{g \in A(\bar{\Omega}) \mid g p=p g$ and $g$ respects all orbits of $H$ in $\bar{\Omega}$.

(c) $H$ is doubly transitive on $\Omega$. In this case the closure of $H$ in the fine (coarse) stabilizer topology on $A(\Omega)(A(\bar{\Omega}))$ is $A(\Omega)$ $(A(\bar{\Omega}))$. The closure of $H$ in the fine stabilizer topology on $A(\bar{\Omega})$ is $K=\{g \in A(\bar{\Omega}) \mid g$ respects all orbits of $H$ in $\bar{\Omega}\}$.

Proof. (c) The first assertion follows from Proposition 4.10 and the $n$-fold transitivity of $H$ on $\Omega$. Let $M$ be the closure of $H$ in the fine stabilizer topology on $A(\bar{\Omega})$. Consider $1<m \in M$ and $\alpha \in \bar{\Omega}$. Since there must be some $h \in H$ such that $m h^{-1} \in H_{\alpha}$ it follows that $(\alpha) m=(\alpha) h$ and that $m \in K$. The equality of $M$ and $K$ may be deduced from the following equivalent formulation of the double transitivity of $H$ on $\Omega$. For every pair of finite sequences $\alpha_{1}<$ $\alpha_{2}<\cdots<\alpha_{n}$ and $\beta_{1}<\beta_{2}<\cdots<\beta_{n}$ of points from $\bar{\Omega}$ such that $\alpha_{i}$ and $\beta_{i}$ share the same $H$ orbit there is some $g \in G$ with $\left(\alpha_{i}\right) g=\beta_{i}$, $1 \leqq i \leqq n$. The verification of part (b) is similar.

We come finally to the continuous representation theorems. The continuous version of Holland's representation theorem [12] follows from Theorem 4.5.

THEOREM 4.13. Suppose $G$ is an $\ell$-subgroup of the t/-group $H$ and that $\left\{P_{\lambda}\right\}$ is the set of topologically closed prime subgroups of $G$. For each $\lambda$ let $\Omega_{\lambda}$ be the chain $G / P_{\lambda}$, let $\theta_{\lambda}: G \rightarrow A\left(\Omega_{\lambda}\right)$ be the usual <-homomorphism with range $G_{\lambda}$, and let $\theta: G \rightarrow \Pi G_{\lambda}$, be the product $\ell$-homomorphism. There is an $\ell$-monomorphism $\hat{\theta}: \mathrm{cl}_{H}(G) \rightarrow$ $\Pi G_{\lambda}^{j}$ extending $\theta$ such that $\hat{\theta}$ is continuous with respect to the $\alpha$-topology on $\Pi G_{\lambda}^{0}$ if and only if $\cap \mathrm{cl}_{H}\left(P_{\lambda}\right)=1$.

Proof. Let $\operatorname{cl}_{H}\left(P_{\lambda}\right)=Q_{\lambda}$, a closed prime subgroup of $K=\operatorname{cl}_{H}(G)$. By Proposition 2.3 every closed prime subgroup of $K$ is a $Q_{\lambda}$ so that $\mathscr{B}\left(\left\{Q_{\lambda}\right\}\right)$ is an $\ell$-topology on $K$ coarser than the original topology by Proposition 2.1. If $\cap Q_{\lambda}=1$ then $\mathscr{B}\left(\left\{Q_{\lambda}\right\}\right)$ is Hausdorff and we may assume it to be the topology on $K$. The existence of $\hat{\theta}$ now follows from standard arguments using Theorem 4.5. The converse follows from Proposition 4.1. 
In light of McCleary's proof that $G^{*}$ is always completely distributive, $G_{\lambda}^{*}$ may be substituted for $G_{\lambda}^{0}$ in the above theorem to obtain a sharper result.

The distinguishing characteristic of a representable $\ell$-group is that the intersection of the conjugates of any prime subgroup is itself prime. Therefore the primes minimal among the topologically closed primes of a representable $t \ell$-group are normal. The next theorem is a generalization of a result of Madell [16].

THEOREM 4.14. Suppose $G$ is a representable 6 -subgroup of the t८-group $H$. Let $\left\{P_{\lambda}\right\}$ be the set of primes minimal among the topologically closed primes of $G$. For each $\lambda$ let $G_{\lambda}$ be the totally ordered group $G / P_{\lambda}$, let $\theta_{\lambda}: G \rightarrow G_{\lambda}$ be the canonical $<$-homomorphism, and let $\theta: G \rightarrow \Pi G_{\lambda}$ be the product $\ell$-homomorphism. There is an $\measuredangle-$-monomorphism $\hat{\theta}: \mathrm{cl}_{H}(G) \rightarrow \Pi G_{\lambda}^{D}$ extending $\theta$ such that $\hat{\theta}$ is continuous with respect to the $\alpha$-topology on $\Pi G_{\lambda}^{D}$ if and only if $\cap \mathrm{cl}_{H}\left(P_{\lambda}\right)=1$.

Proof. Let $Q_{\lambda}$ and $K$ be as in the proof of Theorem 4.13. By Corollary 1.3 each $Q_{\lambda}$ is prime in $K$. As before we may assume the topology on $K$ to be $\mathscr{B}\left(\left\{Q_{\lambda}\right\}\right)$, in which case the existence of $\hat{\theta}$ follows from the remark preceding Proposition 3.5 via standard arguments.

CoRollary 4.15. Suppose $G$ is a totally ordered $\ell$-subgroup of the Hausdorff t/-group $H$. Then there is an $\ell$-monomorphism $\theta: \mathrm{cl}_{H}(G) \rightarrow G^{D}$ over $G$ such that $\theta$ is continuous with respect to the interval topology on $G^{D}$.

We close with the continuous version of the Conrad-HarveyHolland representation theorem for abelian $\ell$-groups ([7], [5]). A subgroup $P_{\lambda}$ is regular if it is prime and if there is another prime subgroup $P^{\lambda}$ minimal among those primes properly containing $P_{\lambda}$. $P^{\lambda} / P_{\lambda}$ is $\ell$-isomorphic to a subgroup of the real numbers whenever it is abelian.

Theorem 4.16. Suppose $G$ is an abelian subgroup of the tל-group $H$ and that $\Gamma=\left\{P_{\lambda}\right\}$ is the set of topologically closed regular subgroups of $G$. For each nonminimal $P_{\lambda} \in \Gamma$ let $\boldsymbol{R}_{\lambda}$ be $P^{\lambda} / P_{\lambda}$ and for each minimal $P_{\lambda} \in \Gamma$ let $\boldsymbol{R}_{\lambda}$ be $\left(P^{\lambda} / P_{\lambda}\right)^{D}$. Let $\theta: G \rightarrow V\left(\Gamma, \boldsymbol{R}_{\lambda}\right)$ be a v-homomorphism ([5]). There is a v-isomorphism $\hat{\theta}: \mathrm{cl}_{H}(G) \rightarrow$ $V\left(\Gamma, \boldsymbol{R}_{\lambda}\right)$ extending $\theta$ such that $\hat{\theta}$ is continuous with respect to the $\alpha$-topology on $V$ if and only if $\cap \operatorname{cl}_{H}\left(P_{\lambda}\right)=1$. 
Proof. Let $Q_{\lambda}$ and $K$ be as above. Propositions 2.1 and 2.3 show that $Q_{\lambda}$ is regular in $K$. The argument then proceeds along the lines of Propositions 4.13 and 4.14.

\section{REFERENCES}

1. R. N. Ball, Convergence and Cauchy structures on lattice ordered groups, preprint, Trans. Amer. Math. Soc., to appear.

2. - $\mathscr{N}$ is not a maximal torsion class, preprint, Boise State University.

3. S. J. Bernau, Varieties of lattice groups are closed under $\mathscr{L}$-completions, Symposia Math., to appear.

4. R. D. Byrd and J. T. Lloyd, Closed subgroups and complete distributivity in latticeordered groups, Math Zeitschr., 101 (1967), 123-130.

5. P. Conrad, Lattice-ordered groups, Lecture Notes, Tulane University, 1970.

6. - The topological completion and the linearly compact hull of an abelian ८-group, Proc. London Math. Soc., (3) 28 (1974), 457-482.

7. P. Conrad, J. Harvey, C. Holland, The Hahn embedding theorem for abelian lattice-ordered groups, Trans. Amer. Math. Soc., 108 (1963), 143-169.

8. L. Fuchs, Partially Ordered Algebraic Systems, Addison-Wesley, 1963.

9. A. M. W. Glass, Ordered Permutation Groups, Bowling Green State University, 1976.

10. A. M. W. Glass, W. C. Holland, and S. H. McCleary, The structure of $\ell$-group varieties, to appear.

11. W. C. Holland, The largest proper variety of lattice-ordered groups, Proc. Amer. Math. Soc., to appear.

12. - The lattice-ordered group of automorphisms of an ordered set, Michigan Math. J., 10 (1963), 399-408.

13. - Outer automorphisms of ordered permutation groups, Proc. Edinburgh Math. Soc., 19 (1975), 331-344.

14. R. L. Madell, Chains which are coset spaces of $t_{\ell}$-groups, Proc. Amer. Math. Soc., 25 (1970), 755-759.

15. Complete distributivity and $\alpha$-congruence, unpublished, Village Community School, 272 West Tenth Street, New York, N. Y., 10014.

16. - Embeddings of topological lattice ordered groups, Trans. Amer. Math. Soc., 146 (1969), 447-455.

17. S. H. McCleary, o-primitive ordered permutation groups, Pacific J. Math., 40 (1972), 349-372.

18. R. H. Redfield, A topology for a lattice-ordered group, Trans. Amer. Math. Soc., 187 (1974), 103-125.

19. B. Šmarda, Some types of topological ८-groups, Publ. Fac. Sci. Univ. J. E. Purkyne (Brno), 507 (1969), 341-352.

20. - Topologies in $\ell$-groups, Arch. Math. (Brno), T. 3 (1967), 69-81.

Received February 3, 1978 and in revised form October 20, 1978.

Boise State University

BoISE, ID 83725 


\section{PACIFIC JOURNAL OF MATHEMATICS}

EDITORS

DONALD BABBITT (Managing Editor)

University of California

Los Angeles, California 90024

Hugo RossI

University of Utah

Salt Lake City, UT 84112

C. C. MOORE and ANDREW OGG

University of California

Berkeley, CA 94720

\section{J. DUGUNDJI}

Department of Mathematics University of Southern California Los Angeles, California 90007

R. Finn and J. Milgram Stanford University

Stanford, California 94305

\section{ASSOCIATE EDITORS}
E. F. BECKENBACH
B. H. Neumann
F. WOLF
K. YoSHIDA

\section{SUPPORTING INSTITUTIONS}

UNIVERSITY OF BRITISH COLUMBIA

CALIFORNIA INSTITUTE OF TECHNOLOGY

UNIVERSITY OF CALIFORNIA

MONTANA STATE UNIVERSITY

UNIVERSITY OF NEVADA, RENO

NEW MEXICO STATE UNIVERSITY

OREGON STATE UNIVERSITY

UNIVERSITY OF OREGON
UNIVERSITY OF SOUTHERN CALIFORNIA

STANFORD UNIVERSITY

UNIVERSITY OF HAWAII

UNIVERSITY OF TOKYO

UNIVERSITY OF UTAH

WASHINGTON STATE UNIVERSITY

UNIVERSITY OF WASHINGTON 


\section{Pacific Journal of Mathematics}

\section{Vol. 83, No. 1 \\ March, 1979}

Richard Neal Ball, Topological lattice-ordered groups ............... 1

Stephen Berman, On the low-dimensional cohomology of some

infinite-dimensional simple Lie algebras .................. 27

R. P. Boas and Gerald Thomas Cargo, Level sets of derivatives ......... 37

James K. Deveney and John Nelson Mordeson, Splitting and modularly

perfect fields......................................

Robert Hugh Gilman and Ronald Mark Solomon, Finite groups with small

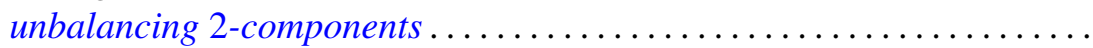

George Grätzer, Andras Hajnal and David C. Kelly, Chain conditions in free products of lattices with infinitary operations..................

Benjamin Rigler Halpern, Periodic points on tori ..................

Dean G. Hoffman and David Anthony Klarner, Sets of integers closed under

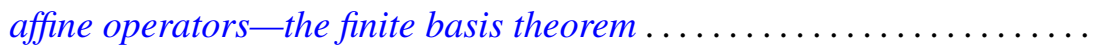

Rudolf-Eberhard Hoffmann, On the sobrification remainder ${ }^{s} X-X \ldots \ldots$

Gerald William Johnson and David Lee Skoug, Scale-invariant

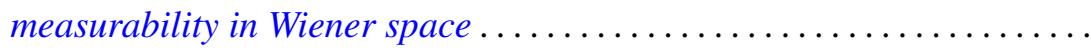

Michael Keisler, Integral representation for elements of the dual of

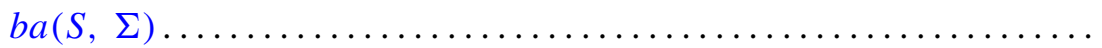

Wayne C. Bell and Michael Keisler, A characterization of the representable Lebesgue decomposition projections ................

Wadi Mahfoud, Comparison theorems for delay differential equations ...

R. Daniel Mauldin, The set of continuous nowhere differentiable functions .

Robert Wilmer Miller and Mark Lawrence Teply, The descending chain condition relative to a torsion theory...

Yoshiomi Nakagami and Colin Eric Sutherland, Takesaki's duality for regular extensions of von Neumann algebras ........ .

William Otis Nowell, Tubular neighborhoods of Hilbert cube manifolds ...

Mohan S. Putcha, Generalization of Lentin's theory of principal solutions of word equations in free semigroups to free product of copies of positive reals under addition

Amitai Regev, A primeness property for central polynomials . ...

Saburou Saitoh, The Rudin kernels on an arbitrary domain. . .

Heinrich Steinlein, Some abstract generalizations of the

Ljusternik-Schnirelmann-Borsuk covering theorem . . . 